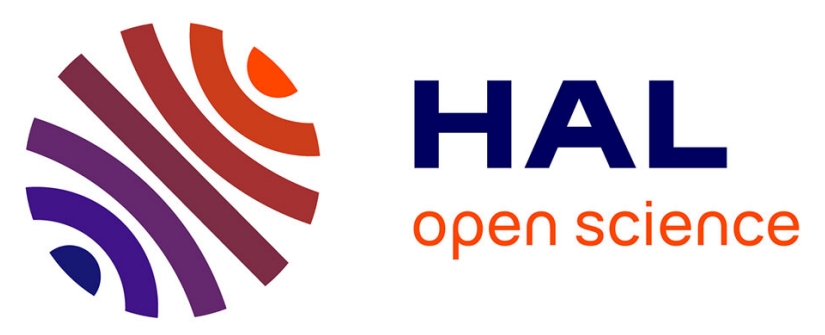

\title{
RATIONALITÉ MANAGÉRIALE ET TRADITION COMPAGNONNIQUE : LA TENSION POLÉMIQUE COMME DYNAMIQUE D'INSTITUTIONNALISATION DANS UN CABINET DE CONSEIL
}

\author{
Jérôme Méric
}

\section{To cite this version:}

Jérôme Méric. RATIONALITÉ MANAGÉRIALE ET TRADITION COMPAGNONNIQUE: LA TENSION POLÉMIQUE COMME DYNAMIQUE D'INSTITUTIONNALISATION DANS UN CAB-

INET DE CONSEIL. Revue management \& avenir, 2009, 10.3917/mav.029.0110 • hal-02153444

\author{
HAL Id: hal-02153444 \\ https://hal.science/hal-02153444
}

Submitted on 9 Dec 2019

HAL is a multi-disciplinary open access archive for the deposit and dissemination of scientific research documents, whether they are published or not. The documents may come from teaching and research institutions in France or abroad, or from public or private research centers.
L'archive ouverte pluridisciplinaire HAL, est destinée au dépôt et à la diffusion de documents scientifiques de niveau recherche, publiés ou non, émanant des établissements d'enseignement et de recherche français ou étrangers, des laboratoires publics ou privés. 


\title{
Rationalité managériale et tradition compagnonnique : la tension polémique comme dynamique d'institutionnalisation dans un cabinet de conseil
}

\author{
Jérôme Méric
}

\section{Résumé}

Autant le management est acquis de longue date au principe érotique de convergence des objectifs, autant la raison contradictoire n'est pas une attitude « naturelle » au milieu gestionnaire. Pourtant l'acceptation de la contradiction porte un éclairage très profitable sur les dynamiques organisationnelles. II en va du cas présenté dans cet article.

L'étude transversale d'une pratique spécifique - l'induction dans un cabinet de conseil - met en évidence un jeu d'interactions entre ses aspects ostensifs (les scénarii d'action), performatifs (les actions) et les artefacts (les productions matérielles) qu'elle produit.

Ces interactions permanentes, loin de susciter un changement, contribuent à maintenir la pratique étudiée dans un état étonnamment immuable. En définitive, ce cas illustre l'institutionnalisation de l'induction dans une organisation spécifique.

L'analyse des résultats fait ressortir trois composantes fondamentales du processus d'institutionnalisation observé. La première est - sans surprise - la répétition (via l'observation ou la prescription). Le deuxième consiste en la conjonction de l'autorité et de l'ancienneté. Enfin, la coexistence - par essence polémique - de scénarii d'action rationnels et d'un mode d'agir traditionnel dans la même organisation contribue à la mutation de la pratique étudiée en institution.

\section{Abstract}

From its early times, management science has always sought ways to implement the erotic principle of goal congruence. In the same time, contradictory Reason seems not to belong to the "natural" environment of managers. This is all the more regrettable that contradiction casts a profitable light onto organizational dynamics. This article introduces a case in which both contradiction and congruence based frameworks need to be developed to fully understand how a specific practice induction in a consulting company - has remained compliant to the same standards for years, whereas the main context and "practices" have changed many times elsewhere. 
The method for research is based on the hypothesis that a practice can be depicted as a set of interactions between ostensive (action scripts), performative (actions) aspects and artefacts (material outcomes).

It appears that these constant interactions, instead of leading to constant change and adaptation, contribute to maintain the studied practice in a - quite surprisingly permanent state. Such interactions can be identified as institutionalizing dynamics.

The research results lead to circumscribe three major components of the observed institutionalization process. The first one is - unsurprisingly - repetition (through observation or prescription). The second one consists in the conjunction of authority and seniority. Finally, the polemic coexistence of rational action scripts and actual traditional actions leads to the change of a simple practice into an institution.

La raison contradictoire (la polémique à son sens étymologique) n'est a priori pas ancrée dans la pratique gestionnaire. Du moins, l'idéal managérial est souvent assimilé au principe érotique de l'obtention de la convergence des efforts dans une direction unique (Fiol, 1991). Dans le même état d'esprit, la notion de dynamique est appréhendée à l'échelle des systèmes entiers, afin de rendre observable et maîtrisable un changement, et non à celle d'une pratique quotidienne, soumise à des interactions permanentes et parfois erratiques. Or ces dernières sont tout autant constitutives d'une dynamique. L'image héraclitéenne du fleuve illustre cette idée de changement permanent sous l'immobilité de la forme globale.

A l'échelle d'une organisation, le changement et la stabilité sont devenus des objets de science, l'adjectif « dynamique » désignant l'aptitude à évoluer ou à provoquer l'évolution (Zollo et Winter, 2002). Ces "modèles d'action collective appris et stabilisés" (op. cit., p. 340) sont censés permettre la conception et la direction du changement des routines de l'organisation dans une direction souhaitée, comme autant de moteurs susceptibles de mouvoir tout ou partie du système (Cyert \& March, 1963, Levitt \& March, 1988, Nonaka \& Takeuchi, 1995). Cette vision à la fois macroscopique et unidirectionnelle permet tout d'abord d'assimiler dynamique et mouvement. Par ailleurs, elle présente l'avantage de fournir une opposition, aux apparences tout aristotéliciennes, entre la dynamique et l'immobilité.

L'acceptation de la contradiction (enantiodromie) porte au contraire à poser un regard tout autre sur les dynamiques organisationnelles. Elle conduit notamment à considérer que ces dernières peuvent échapper à toute forme d'intention rationnelle, et qu'elles engendrent aussi bien l'immobilité globale que le changement. March, avec Simon (1958), suggère l'association du terme de routine avec celui d'inertie de l'organisation. C'est pourtant ce même auteur qui décrit les routines comme un facteur de changement majeur, à travers des schémas d'adaptation inspirés de la cybernétique (Cyert et March, 
1963), ou des interactions soutenant l'apprentissage (Levitt et March, 1988, Feldman, 2000) et l'innovation (Miner, 1990). A considérer l'évolution des théories à ce sujet, il semble que la contradiction se soit résolue par la mise en valeur de ces dernières approches, et l'abandon de l'association entre routine et immobilité. Rares sont ceux qui, au contraire, ne cherchent pas à résoudre le conflit, et font l'hypothèse que changement et stabilité proviennent des mêmes dynamiques (Feldman, 2003).

Dans cet article, nous proposons d'appréhender le cas d'une organisation par les pratiques qu'elle met en œuvre. Plus précisément, nous examinons ce que les anglo-saxons désignent par l' " induction », c'est-à-dire le processus par lequel les nouveaux venus sont accueillis puis intégrés dans la structure. Cette pratique a été étudiée - avec d'autres - dans le cadre d'un cabinet de conseil, pour laquelle elle revêt une importance capitale. Dans une organisation de cette nature, l'induction apparaît comme un vecteur de changement majeur. C'est la raison pour laquelle elle a fait l'objet d'une attention toute particulière.

L'étude de cas présentée est longitudinale, fondée sur des observations ponctuelles (et réitérées), et des entretiens à grande échelle. Pendant la période d'investigation, aucun changement majeur n'a été observé, même lorsqu'il aurait pu sembler nécessaire. Ces résultats de recherche nous amènent à considérer la contradiction qui peut ici se manifester entre la rationalité érigée en principe de fonctionnement par le cabinet, et l'adoption patente d'un mode d'agir purement traditionnel. Pour ce faire, nous étudions les dynamiques induites entre les artefacts, les éléments ostensifs et performatifs de la pratique d'induction. Nous montrons comment de constantes interactions parviennent à immobiliser l'organisation. Ce jeu polémique entre la tradition observable et la rationalité managériale érigée en référence absolue ne serait-il pas en fin de compte significatif d'un processus d'institutionnalisation particulier?

Dans un premier temps, nous présenterons la méthodologie mise en œuvre pour l'étude de cas, ainsi que le cadre théorique qui a guidé tant la méthodologie que l'interprétation des résultats. Ensuite, les principales observations sont présentées, afin de souligner leur caractère paradoxal. Enfin, l'interprétation des résultats est fournie, pour aboutir à des propositions relatives au phénomène d'institutionnalisation dans la sphère gestionnaire.

\section{Méthodologie de la recherche : une étude de cas concentrée sur la pratique}

L'objet de la présente recherche étant initialement l'étude de pratiques, il a semblé nécessaire de les définir avant de pouvoir déterminer la méthode à mettre en œuvre pour l'analyser.

\subsection{Qu'appelle-t-on une pratique?}


On compte au moins deux approches de ce que peut être une pratique. La première, que l'on peut décrire comme issue de l'école " rationaliste », définit une pratique comme un ensemble déterminé et stable de tâches élémentaires ou d'actions menées par des " acteurs ». Selon ce cadre d'analyse, le changement dans les pratiques et l'apprentissage peuvent être appréhendés comme des actes rationnels et délibérés mis en œuvre dans une durée déterminée (Zollo et Winter, 2002). Cette définition présente l'avantage de la simplicité, de l'observabilité, et mène assez naturellement à des descriptions fondées sur les méthodologies traditionnelles d'analyse de processus ${ }^{27}$

Après une brève phase exploratoire en entreprise, il apparaît à chaque fois que cette définition se réfère plus à ce que l'on appelle communément des " routines » qu'à des pratiques (au sens étymologique du terme). Les pratiques semblent inclure non seulement des tâches organisées selon des scénarii d'action, mais elles incorporent aussi l'action pour elle-même. La pratique associe des modèles d'action et la manière dont ces modèles sont effectivement appliqués (Bourdieu, 1977). De fait, le terme grec praxis décrit l'action et la pensée au même moment. Plus précisément, il désigne l'action comme contexte de la pensée et la pensée comme contexte de l'action (Castoriadis, 1998). Si l'on souscrit à cette acception, on doit élargir l'approche de ce qu'une pratique peut être, en tenant compte du langage, des productions matérielles, des intentions et des réalisations. Le sujet « pratiquant », selon Castoriadis (1998) est à son tour transformé par sa propre expérience. Cette seconde approche confère à la pratique une autonomie que l'école rationaliste ne lui octroie pas : une pratique est source de changement, elle n'en est pas seulement l'objet ; elle porte cette contradiction en elle. En ce qui concerne l'apprentissage, les pratiques vues comme praxis ne nécessitent pas l'« invention » de forces exogènes susceptibles de les faire évoluer comme l'intention formelle, des « méta-pratiques » ou d'autres artefacts. Cette définition rassemble de nombreuses sous-approches, comme celles fondées sur le constructivisme ou la « structuration » (Giddens, 1984, Latour, 1987). Cette seconde sous-approche, qui peut être présentée comme l' " école de la structuration et/ou de la traduction ", nous porte à considérer les pratiques comme un agrégat d'éléments ostensifs (modèles d'action explicités dans un discours), performatifs (les actions effectivement entreprises) et d'artefacts (outils et productions matérielles), mis en œuvre dans ou par des " actants ", et en constante interaction. Selon ce cadre conceptuel, l'apprentissage est le fait d'une dynamique permanente et ne trouve pas nécessairement son origine dans l'intention ou la rationalité (Pentland et Feldman, 2005).

Le choix de cette seconde approche implique l'adoption d'une méthodologie spécifique qu'il convient de détailler. 


\subsection{Durée, détail, et singularité : le choix de l'étude de cas}

Lorsque les terrains ou les préoccupations de recherche sont encore peu explorés, il semble nécessaire de valoriser dans un premier temps des études approfondies et si possible menées dans la durée. L'apport des études de cas peut consister à cerner plus précisément des objets encore flous (Glaser \& Strauss, 1967 ; Eisenhardt, 1989).

En second lieu, l'étude de cas peut être préférée à une appréhension quantifiée à plus large échelle d'observations lorsque ce que l'on étudie - en particulier une pratique - est difficilement mesurable (Strauss \& Corbin, 1990 ; Yin, 1994). En cela, l'étude de cas permet d'opérationnaliser des objets complexes pour des recherches ultérieures.

Enfin, une étude de cas offre l'occasion non seulement d'appréhender dans son entièreté le phénomène observé, mais aussi de percevoir des dynamiques qu'une enquête ne permettrait pas de saisir (Yin, 1994).

Pour l'ensemble de ces raisons, le recours à une étude de cas se justifie pour étudier l'évolution (ou l'absence d'évolution) d'une pratique dans un secteur particulier, et qui plus est dans la durée.

\subsection{Méthode de recherche}

Dans un premier temps, une phase exploratoire a été mise en œuvre. Cette étape a permis de cerner les " contours" de la pratique étudiée. Par la suite, des entretiens et des observations ciblés sur la pratique en question ont été menés. Les entretiens ont permis de rencontrer des associés, des consultants à différents grades d'ancienneté, et la seule personne chargée du recrutement et de l'administration des ressources humaines. Les entretiens ont été renouvelés autant de fois qu'il semblait nécessaire. La petite taille de la structure a très vite soulevé des problèmes de disponibilité des interlocuteurs. Nous avons aussi collecté des documents privés et publics. Les derniers correspondaient essentiellement à des sites internet et à des articles de presse consacrés à l'entreprise. Les documents privés se sont faits plus rares, non en raison de la confidentialité, mais plutôt parce que la hiérarchie plate du cabinet favorise la communication directe. Nous avons toutefois pu consulter des notes de service en rapport avec le processus d'induction, puis des mails et des programmes de formation continue suivis par le personnel. Le tableau 1 fait la synthèse de ces documents, classés selon qu'ils alimentent des données ostensives, performatives, et des bases d'artefacts (en relation avec la définition de la pratique précédemment évoquée). 
Aspects ostensifs de la pratique (entretiens)

Associés
Consultants récemment embauchés Consultant junior
Consultant senior
Personnel administratif
Observations
Entretiens de recrutement (rounds) Entretiens de feedback et d'évaluation

Artefacts

Documents internes (notes de service, agendas, programmes de formation continue...)

Documents externes (sites web, articles dans la presse professionnelle spécialisée...)

Tableau 1 - Démarche de recueil de données pour l'étude de cas

L'exploitation des données et la méthode d'analyse se sont progressivement construites selon les informations qui pouvaient être collectées au moment. En définitive, les premières investigations ont essentiellement fait remonter des éléments d'artefacts et ostensifs de la pratique étudiée. Dans l'optique de mieux cibler les recherches ultérieures, ces premières données ont été exploitées pour établir les « frontières » de la pratique d'induction. II s'est agi non pas d'établir une nomenclature de tâches rattachables à cette dernière, mais seulement d'identifier les « actions-clés » par lesquelles nous pouvons considérer que l'induction est mise en œuvre. Ces actions-clés peuvent être hiérarchisées et présentées comme la figure 1 le suggère.

Processus d'induction (complet)

Recrutement

Induction

Gestion des missions Formation

Feedback

Evaluation

Figure 1 - "actions-clés" ostensives : frontières possibles pour la pratique d'induction

Le choix a été fait d'incorporer le recrutement dans le processus d'induction, car il est apparu que le cabinet de conseil en question associe systématiquement les deux processus en vue de l'intégration du personnel, et des consultants en particulier. Ces " actions-clés » ont constitué le centre de nos investigations, un guide pour nos entretiens et les phases d'observation, mais ils n'ont pas pour autant été l'objet exclusif de ces dernières. Le contexte de leur mise en œuvre appartient au champ de la pratique, et nécessite autant d'attention. 
La démarche d'analyse des informations recueillies a suivi deux directions. La première, consistant à formaliser au mieux les processus, a vite débouché sur l'impasse de l'absence de changement apparent. La deuxième a consisté à mettre en relation les éléments ostensifs, performatifs et les artefacts pour évaluer leurs interactions et l'impact de ces dernières sur la pratique étudiée.

Les phases d'observation et les artefacts ont confirmé dans une large mesure les scénarii d'action identifiés (ie les éléments ostensifs). Ensuite, l'étude des interactions nous a permis d'examiner comment les trois éléments constitutifs de la pratique parviennent à se maintenir les uns les autres et finalement conserver le système en l'état. Nous avons ensuite essayé de comprendre comment une organisation de la sorte parvient à induire une si forte standardisation de ses pratiques. C'est alors que nous avons pu souligner l'attachement organisationnel aux valeurs, à la tradition, et à la cooptation.

\section{L'étude de cas : pratique de l'induction dans un cabinet de conseil}

Nous suivrons ici pas à pas notre démarche d'interprétation, afin d'expliciter pleinement la méthodologie mise en œuvre. Après une brève présentation du cas, les éléments ostensifs sont étudiés. Leur relation aux artefacts, en raison de leur forte interaction auto-confirmatoire est ensuite présentée. Dans un troisième temps, la norme de fonctionnement de l'induction, telle qu'elle ressort de l'interaction des artefacts et des éléments ostensifs est explicitée. Enfin, la triangulation s'achève par l'examen des aspects performatifs.

\subsection{Informations générales sur le cabinet de conseil}

Le cabinet étudié est spécialisé dans le conseil en stratégie d'entreprise. Sa création est due à l'association d'anciens consultants de Mc Kinsey, Booz Allen, et BCG. Le mode de recrutement adopté est une réplique de celui mis en œuvre par ces entreprises "d'origine ». Aux dires des interlocuteurs rencontrés, l'objectif poursuivi par ces consultants était de travailler dans une structure plus petite et surtout ne consacrer l'essentiel des missions qu'à la stratégie. Aujourd'hui, le bureau parisien du cabinet fonctionne avec 10 associés et 50 consultants. En raison de sa petite taille, la hiérarchie qu'il adopte est plate (ie à deux niveaux). Les associés savent se rendre disponibles pour tous les consultants, et répondre à leurs questions à tout moment.

Les principaux clients du cabinet sont les dirigeants de grandes entreprises internationales, ou de plus modestes intervenant dans des secteurs dynamiques. L'offre du cabinet consiste essentiellement en du conseil stratégique. L'opportunité de se diversifier dans l'analyse de processus a toujours été rejetée par les associés depuis la création de l'entreprise. 
La méthode de travail du cabinet relève du " sur mesure ». Les associés préfèrent concevoir des méthodes propres à chaque client plutôt qu'adapter des méthodes déjà prêtes à des situations singulières. Cette dernière attitude est d'ailleurs vécue en interne comme la marque d'une absence d'éthique professionnelle. Les clients sont impliqués de façon croissante au fur et à mesure que la mission avance, de manière à pouvoir prendre en main la solution livrée dès avant le départ des consultants. Cette démarche est reconnue en interne comme un " facteur-clé de succès » du cabinet. L'exigence en adaptabilité et en capacité d'analyse des consultants est d'autant plus élevée.

Après quatre années difficiles, le cabinet de conseil connaît une nouvelle période de prospérité à partir de 2005. Pour cette raison, le recrutement et l'induction sont largement réactivés, du moins jusqu'en 2008.

\subsection{Les données ostensives (première phase d'entretiens) : le paradoxe d'un formalisme informel}

II n'est pas surprenant que, dans un cabinet de conseil, une pratique quelle qu'elle soit puisse être présentée par les personnes interrogées comme un processus. En revanche, deux aspects ostensifs relatifs à cette pratique doivent être relevés, car ils se révèlent contradictoires. Tout d'abord, l'induction est décrite par tous les interlocuteurs comme un processus parfaitement normalisé, alors qu'aucune règle écrite n'existe à ce sujet (aucun artefact ne dicte cette manière de faire, ni les étapes à franchir). De plus, il peut paraître surprenant que des descriptions aussi cohérentes et formalisées de l'induction se manifestent dans une organisation où les relations informelles entre les membres sont mises en avant. Les associés sont particulièrement attachés à une nette différenciation des étapes du processus d'induction. Très vite après les premiers entretiens, il a été possible de concevoir un schéma de flux pour représenter le processus, lequel schéma présenté ensuite aux interlocuteurs a fait l'objet d'un consensus complet. La figure 1 restitue ce que, dans le métier, on qualifie de flow chart pour l'induction.

Cette première phase d'entretiens suggère l'acceptation formelle et informelle (via le discours et les représentations) d'une tradition de l'induction, la répétition consensuelle, non-écrite et très formalisée d'une " manière de faire ».

\subsection{La confrontation aux artefacts : la confirmation du respect de la règle et de son succès}

L'absence de manuel de procédure ou de document de cet ordre dans le cabinet ne doit pas obérer le fait que d'autres artefacts confortent le point de vue restitué par les éléments ostensifs. Le récit des consultants concernant leur propre embauche " suit à la lettre » ce qui apparaît dans les pages 
dédiées du site web du cabinet et dans les articles destinés notamment aux jeunes diplômés. II s'agit bien là de la manifestation du respect d'une procédure non écrite. L'annonce de cette procédure dans le site et les journaux spécialisés contribue à son tour à ce que la norme soit respectée. Les documents internes, quoique rares, corroborent le respect des scénarii d'action. Les agendas pour leur part, peuvent être considérés comme des artefacts (ils ne relèvent pas d'une observation directe), mais aussi comme des aspects performatifs de la pratique (ils sont autant de " preuves " que les règles sont respectées). Ce sont les documents externes qui, véhiculant notamment un message à l'attention des candidats, exercent une influence coercitive sur la pratique interne. Dans la plupart de ces supports, les journalistes insistent sur le rôle du recrutement, " la méthode pour construire une équipe efficace et talentueuse ", "le pourquoi d'un turnover aussi faible si on le compare à la moyenne du secteur ». Non seulement de telles productions incitent au respect de la règle (elles la rendent explicite et publique), mais elles fournissent aussi un miroir " autoréalisateur » par la preuve qu'elles fournissent de son succès. Cette interaction des composantes de la pratique est restituée dans la figure 2 .

\subsection{L'explicitation de la règle restituée par les artéfacts et les éléments ostensifs : formalisme, loyauté, et élitisme}

Du recrutement aux premières promotions, le processus d'induction mis en œuvre dans le cabinet semble très formalisé, sans pour autant être inscrit dans le moindre manuel de procédures. La norme qui ressort des entretiens et de la recherche documentaire peut être décrite comme privilégiant un formalisme particulier, la loyauté (ou peut-être la fidélité), et l'élitisme. Elle tient en sept points. Tout d'abord le recrutement est très ciblé (1), respectant un cahier des charges strict (2), et suit un processus très formalisé (3). Une fois les consultants recrutés, le feedback mis en œuvre pendant les missions respecte un ensemble de règles précises (4). Les programmes de formation suivent une démarche systématique (5). Enfin, l'induction semble conçue pour privilégier une " expertise généraliste » (6) et minimiser le turnover des consultants (7).

\section{(1) Un recrutement ciblé}

Tous les consultants juniors sont diplômés des meilleures écoles d'ingénieurs et de commerce françaises (Polytechniques, Centrale, Les Mines, Les Ponts, HEC, ESSEC, ESCP). Ce choix très élitiste est considéré devant garantir la possibilité de faire face à l'exercice d'un métier " très exigeant ». Un associé souligne que « la diversité n'est pas vraiment l'objectif ». En définitive, l'intégration et la complicité entre consultants est aussi recherchée comme résultat d'un esprit de corps.

(2) Des compétences précises recherchées 
Les qualifications des consultants ne sont formalisées ni en terme de connaissance ni en termes techniques. Le processus de recrutement se concentre sur l'identification des comportements au travail et de la capacité d'analyse. Les candidats sont évalués au regard de "benchmarks ». Ces archétypes comportementaux peuvent être restitués par n'importe lequel des consultants, mais, une fois de plus, ils ne sont pas écrits, à l'exception d'une phrase notée sur le site web : «nous recherchons des personnes qui savent analyser des situations complexes et les traduire en termes simples dans peu de phrases ».

\section{(3) Processus de recrutement très formalisé}

Les jeunes diplômés doivent suivre 3 séries de 2 entretiens chacune. Les critères de recrutement pris en compte sont de trois ordres :

- la motivation, « pas si importante, car ceux qui s'apprêtent à suivre un processus aussi lourd sont nécessairement motivés » (un associé) ; - l'expérience ou plutôt la capacité à apprendre de cette dernière ; " Maintenant, je sais parfaitement qu'ils n'avaient rien à faire de mon année de césure réalisée chez Saint Gobain », affirme un junior, " ce qui était important, c'étaient les enseignements que j'en avais tirés " ;

- les compétences spécifiques reposent sur le sens de l'analyse (comme nous l'avons déjà évoqué) et la capacité de restitution synthétique.

Le comportement et l'attitude sont pris en compte au regard des standards déjà mentionnés, mais ils demeurent des variables secondaires, l'expérience pouvant les faire évoluer. Chaque entretien contient une brève étude de cas, pendant laquelle la capacité à faire face à la situation est testée. Les connaissances techniques sont d'autant moins valorisées que les candidats n'ont pas nécessairement de cursus gestionnaire (les ingénieurs en l'occurrence). Après chaque interview, les recruteurs notent le candidat. Les diplômés qui ne se voient opposer aucun veto (une possibilité d'émission est accordée à tous les consultants), qui obtiennent au moins une évaluation « enthousiaste ", et moins de deux « non » de la part des recruteurs sont embauchés. "Dès que vous êtes entré, on vous parle de la règle des « deux non ». Mais vous ne savez jamais si quelqu'un dans l'équipe s'est opposé à votre recrutement » (un consultant junior).

(4) Un feedback répondant à des règles précises

Le système de feedback fait partie de celui plus large consacré à l'évaluation des consultants. II suit deux processus distincts et complémentaires. D'abord, chaque mission suscite des feedbacks individuels (au moins un pendant la mission, et un autre à son terme). Ces retours sont résumés dans ce que l'on appelle " la synthèse semestrielle ». Après chacun de ces entretiens individuels, les associés et les chefs de mission se réunissent pour déterminer 
si le consultant doit progresser dans la structure ou en sortir (il s'agit de la règle du up or out).

(5) Une démarche de formation systématique.

Les programmes de formation sont dispensés pour la plupart hors de la structure, mais sont conçus pour répondre à un cahier des charges précis. $\mathrm{A}$ l'exception des cas où une mission requiert un syllabus spécifique, les programmes de formation sont prédéterminés pour chaque niveau hiérarchique. Par exemple, les consultants juniors suivent tous un séminaire de « finance avancée », alors que les associés se concentrent sur le leadership et le développement personnel (« comment adapter votre style de leadership à des attitudes au travail divergentes » est le titre de l'un de ces programmes).

(6) La recherche d'une " expertise généraliste ».

La spécialisation n'est constatable qu'à partir d'un certain niveau d'ancienneté. Personne n'est censé se spécialiser avant quatre ou cinq ans d'expérience dans le cabinet. Les consultants sont censés rester très polyvalents tout au long de leur carrière. Le cabinet se positionne sur le marché du conseil en stratégie, ce qui explique une telle approche. Ce choix est aussi cohérent avec la taille de l'entreprise : " plus vous êtes petit, plus vous devez être polyvalent pour répondre à une demande extrêmement variée » (un associé). Si le contraire devait se produire, une sur-spécialisation soulèverait des problèmes de gestion du personnel insurmontables.

(7) Un turnover très faible.

Le turnover des consultants est l'un des plus faibles du secteur. Cette information est confirmée dans la presse professionnelle. Parmi 60 employés, 4 consultants quittent la société chaque année pour contribution insuffisante. Les autres départs constatés (moins d'un par an) correspondent à des personnes embauchées par un client, ou à des femmes suivant des maris mutés.

\subsection{La triangulation par les aspects performatifs : la dynamique et l'immuabilité}

Les pratiques observées convergent avec les aspects ostensifs évoqués jusqu'à présent, et ce à chaque étape du processus décrit en figure 1 bis. Elles apportent aussi un éclairage complémentaire sur la manière dont certaines de ces étapes sont mises en œuvre par le personnel de l'entreprise. Cette analyse permet de comprendre en particulier comment la pratique d'induction se maintient (et se reproduit) par l'interaction permanente de faits, d'actions, et de discours. Tous les changements qui ont pu être relevés sur la période relèvent d'adaptations minimes appliquées à une règle qui apparaît comme immuable. II s'agit pour l'essentiel de modulations de faible ampleur dues à la 
personnalité du candidat, à l'urgence d'un recrutement, et autant de cas particuliers. Toutefois, la « loi commune » n'est nullement remise en cause par ces faibles embardées (par exemple, le nombre d'entretiens ne sera pas réduit pour quelque raison que ce soit).

Nous examinons à présent l'ensemble de ces enseignements : tout d'abord, la mise en évidence d'un conditionnement préalable aux entretiens de recrutement (un rituel non évoqué dans les entretiens), puis le retour sur le vécu des entretiens par les candidats, et enfin la délibération des recruteurs.

\section{(1) Le conditionnement préalable à l'entretien.}

« 10 heures du matin. La candidate entre pour sa première série d'entretiens. Elle se présente au bureau d'accueil, demande à rencontrer Monsieur $\mathrm{T}$. (associé). Elle attend 15 minutes assise en face du bureau. Enfin Monsieur T. arrive. Ils se serrent la main. II lui demande d'attendre encore une minute pour aller chercher le consultant qui doit participer à l'entretien (un entretien se fait à deux consultants au minimum). Les locaux sont répartis sur deux étages. Le rez-de- chaussée rassemble l'accueil et les bureaux. Les salles se réunion sont à l'étage. C'est pourquoi la candidate ne voit qu'une partie (l'accueil) de l'endroit où elle travaillera. L'associé, le consultant et le candidat montent au premier étage ». Bien que ce récit puisse paraître relater des actes extrêmement simples et spontanés, la séquence qu'il suit est répétée de la même manière aux entretiens suivants. Bien que nous ne disposions pas d'éléments qui nous permettent d'affirmer que ce rituel est accompli de manière volontaire, le processus place le candidat dans un contexte préinitiatique, par la conjonction d'une longue attente et d'une organisation spécifique de cette dernière.

\section{(2) Les entretiens.}

Bien entendu, les entretiens diffèrent ne serait-ce qu'en raison du caractère des recruteurs et des candidats. Néanmoins les standards précédemment évoqués sont amplement respectés. Pour chaque entretien, les recruteurs s'assoient d'un côté d'une longue table de réunion. Le candidat est seul sur " l'autre rive ». Le premier entretien porte essentiellement sur la motivation et l'expérience. Lorsque le candidat a tendance à aborder des propos purement techniques, il est aimablement appelé à rectifier sa position au plus vite. Les questions sont adaptées à chaque candidat, mais elles tendent vers les mêmes objectifs que sont la mesure de l'autonomie et l'appréciation des capacités analytiques. Le deuxième entretien est concentré sur des études de cas, et suit une démarche ritualisée. Après un bref exposé introductif réalisé par l'associé ou le consultant, le candidat se voit présenter une situation oralement tout d'abord, puis avec l'aide d'un support documentaire. On lui octroie deux minutes pour réfléchir à la réponse. Après cela, les recruteurs font en sorte d'élargir le cadre de réflexion du candidat (à d'autres contextes, par exemple), et demandent des réponses les plus brèves possibles. Si le 
candidat ne saisit pas immédiatement la problématique du cas, il ne reçoit aucune information complémentaire ; les recruteurs peuvent alors proposer un autre sujet.

\section{(3) les délibérations}

Tous les associés participent à la délibération. S'y ajoutent les consultants qui ont pris part aux entretiens. Chaque recruteur donne son avis sur le candidat et lui attribue une note. A l'occasion d'une réunion, nous avons pu assister au cas où un associé et un consultant (sur dix personnes ayant rencontré le candidat) ont exprimé leur réticence à recruter un candidat. La règle des « deux non » appelait le rejet de la candidature. Pourtant, en raison du démarrage d'une mission importante dans les deux semaines, un recrutement immédiat paraissait indispensable. Pour cette raison, les deux participants « hostiles » ont dû étayer leurs points de vue, et finalement ont reconnu que leur opinion était plutôt fondée sur un « relationnel difficile de prime abord » que sur d'autres éléments « tangibles ». Ils ont alors retiré leur note négative. La majorité semble alors avoir imposé sa vision à deux individus. Toutefois, la réunion s'est toujours déroulée de manière à ce que l'on ne déroge par à la règle des " deux non », le respect de cette dernière étant apparu comme une priorité.

L'analyse des aspects performatifs et ostensifs montre que ces deux faces de la pratique interagissent constamment, non pour favoriser un changement d'ordre quelconque, mais pour maintenir le système en l'état. Ces interactions se révèlent de natures distinctes et complémentaires. II apparaît qu'elles relèvent de processus mimétiques, confortés par l'exercice d'une autorité fondée sur l'ancienneté, le recours à la rationalité ostensive et enfin l'usage de la contre- exemplarité.

\section{La reproduction mimétique de l'induction}

De fait, chaque étape du processus décrit dans les scénarii d'action est scrupuleusement respectée par les individus. A l'inverse, on n'apprend pas ces pratiques aux nouveaux venus, du moins pas de manière formelle et réfléchie. Ces derniers acquièrent la pratique "sur le tas », en reproduisant ce qu'ils observent. Ce mode d'interaction contribue à maintenir l'induction dans un état stable, pour ne pas dire immuable. Les nouveaux-venus restituent un scénario d'action dont on peut observer la réalisation, et agiront dans le futur conformément à ce dernier. Celui qui apprend à pratiquer "l'induction » dans ce cabinet entre dans une "communauté de pratiques", au sens de Wenger et Snyder (2000) : "Les communautés de pratiques sont des groupes de personnes qui partagent un intérêt ou une passion pour quelque chose qu'ils font et apprennent à faire de mieux en mieux au fur par leur interaction régulière ${ }^{28} »$. Une telle construction, comme le soulignent Wenger et Snyder, ne requiert aucune forme d'intentionnalité. Les nouveaux-venus développent les schèmes de l'induction sans nécessairement le vouloir, ni poursuivre un 
quelconque objectif. Ils reproduisent ce qu'ils ont vécu comme candidats, puis " de l'autre côté », comme consultants recruteurs. Le mimétisme est reconnu comme un moyen d'apprentissage : « Passer au peloton d'exécution avant de passer dans le peloton. Rien de mieux pour apprendre à manager un entretien d'évaluation! " (un consultant sénior). La reproduction n'est cependant pas le seul mode d'interaction des aspects ostensifs et performatifs.

\section{L'autorité fondée sur l'ancienneté}

L'autorité assure aussi le respect des scénarii d'action. La taille de l'entreprise ne permet pas la traduction de cette dernière en ordres formels et en objectifs. Elle devient visible lorsque les consultants ne respectent plus les normes internes : "Tu ne devrais pas autant aider le candidat. N'oublie pas que nous n'avons pas du tout fait la même chose avec toi » (un associé à un consultant junior lors de son premier entretien de recrutement) ; "La prochaine fois, tu n'oublies pas de m'appeler avant d'accueillir le candidat, OK ? " (un associé à une consultant avant un entretien). L'influence de l'autorité sur le mimétisme et l'obéissance est renforcée par la rencontre d'un système de valeurs internes et d'un souci constant d'action rationnelle. Nous avons pu relever au moins deux valeurs promues par le cabinet en relation avec l'induction. Tout d'abord, le respect des règles et du groupe est érigé en principe : " Up or out » signifie aussi « tu es avec nous ou pas » (un consultant sénior). En second lieu, la légitimité (et donc l'autorité) provient directement de l'ancienneté dans le structure : "Bien sûr, vous pouvez toujours discuter avec un associé ou un sénior, mais c'est toujours le plus ancien dans une réunion qui a le dernier mot » (un consultant junior). Du fait que la règle du « up or out » s'applique dans le cabinet, il semble logique que l'ancienneté traduise la légitimité. Ce phénomène trouve bien sûr sa justification rationnelle .

\section{La rationalité ostensive et le recours au contrexemple}

L'autonomie laissée aux consultants est supposée accroître l'efficacité et l'efficience dans la gestion des missions : « Nous n'avons pas de temps à perdre avec des personnes qui peuvent un jour devenir d'excellents consultants " (un associé). Les observations et les témoignages concourent à cette rationalisation, comme autant de preuves que les règles et les procédures non écrites sont fondées sur le bon sens et la justesse de raisonnement. « En ce qui me concerne, je n'aurais pas été capable de réussir dans cette mission si je n'avais pas suivi la formation en finance approfondie. Je l'avais tellement peu vu avant, ou du moins c'était superficiel » (un consultant junior - ingénieur).

Nous avons aussi noté le recours aux « histoires de ce que tu ne devrais jamais faire », construites à l'instar des fables ésopiques. Lorsqu'il est demandé aux consultants de justifier pourquoi le récit qu'ils nous font relève du contrexemple, nous recueillons une réponse rationnelle. Si nous ne formulons pas cette demande, le contrexemple est livré tel quel, sans la 
moindre rationalisation, comme " la chose que l'on ne devrait jamais faire ". Cette dualité dans l'attitude de nos interlocuteurs permet de déceler à quel point les comportements demeurent ancrés dans des traditions et des valeurs.

L'ensemble de ces résultats intermédiaires nous conduit à formuler l'hypothèse suivante : les constantes interactions entre les aspects performatifs et ostensifs de l'induction concourent à maintenir cette pratique aussi proche que possible d'un standard, à l'image de ce qui se produit dans un système autopoïétique. L'analyse de ces interactions est proposée dans la figure 3 , et une synthèse pour l'ensemble de la pratique (artefacts compris) en figure 3 bis. Tous les éléments analysés contribuent à la construction d'une communauté de pratique, comme nous l'avons déjà mentionné. Toutefois, si l'on se réfère à Wenger et Snyder, de telles communautés suivent des modèles particuliers, et il convient de s'interroger sur le modèle prévalant dans ce cabinet de conseil.

Figure 3: L'induction comme pratique auto-entretenue - interaction des aspects ostensifs et performatifs

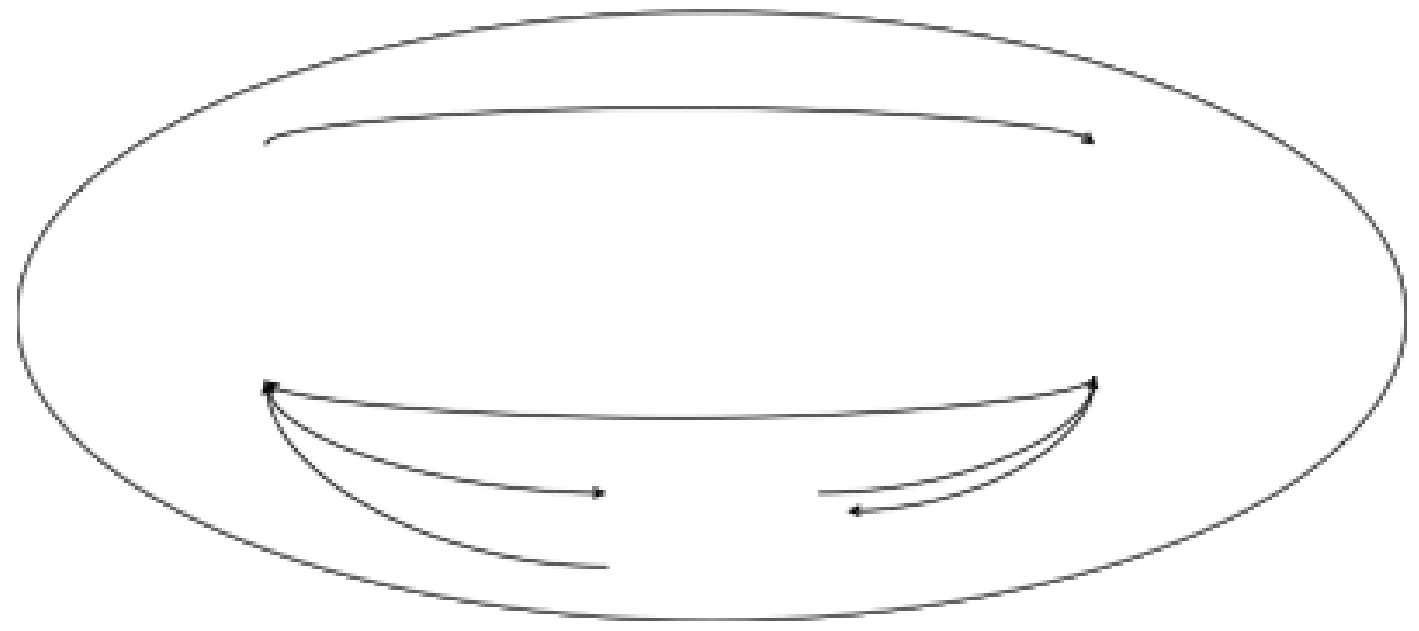

Figure 3bis: l'induction comme une pratique auto-entretenue et ritualisée

\section{L'interprétation des résultats : mécanismes d'institutionnalisation d'une pratique}

Comment une pratique, en l'occurrence l'induction, peut-elle être érigée en loi commune pour l'ensemble des membres d'une organisation ${ }^{29}$ ? Cette étude de cas offre, par la mise en œuvre d'une méthodologie spécifique, la possibilité de répondre à cette question dans une situation spécifique. La notion de « loi commune » nous a été suggérée par le constat d'un premier paradoxe, lié au formalisme strict des démarches dans un univers qui se veut relativement informel, et où les règles ne font presque jamais l'objet d'un écrit. Là où les procédures ne sont pas consignées dans un manuel, elles semblent gravées dans l'esprit de chacun. Non seulement la démarche d'induction, mais aussi 
les aspects qualitatifs tels les critères de recrutement font l'objet d'un consensus que nous n'avons pas pu mettre en défaut. Nous analyserons d'abord les modalités du consensus présent dans le cabinet de conseil étudié, puis nous proposerons une synthèse sur les mécanismes d'institutionnalisation relevés dans cette recherche.

\subsection{L'étude de cas : une organisation fondée sur l'agir traditionnel et en valeur}

Le « formalisme informel » tel que nous l'avons qualifié précédemment trouve avant tout sa source dans la «norme sectorielle ». C'est là un argument avancé par les associés. Tous les fondateurs ont d'abord travaillé dans des grands cabinets de conseil (Mc Kinsey pour la majorité d'entre eux). Cette expérience commune contribue certainement à la formation des processus que nous avons étudiés. Néanmoins, il est difficile de comprendre l'absence de changement par la simple reproduction de « ce qui se fait dans le secteur ».

C'est à ce stade qu'intervient la question du contrôle des membres du cabinet.

Les démarches formelles de feedback et d'évaluation ne constituent qu'une partie de ce dernier. L'étude de cas révèle, notamment par les aspects ostensifs, l'existence de valeurs partagées. Le recours fréquent aux contrexemples, ou encore l'importance accordée à l'ancienneté dénotent aussi un attachement à des valeurs et à une forme d'action traditionnelle. Alors que le terme de " coaching » est aujourd'hui très prisé dans le milieu professionnel, les membres du cabinet lui préfèrent unanimement celui de « compagnonnage ». L'emploi de ce terme a bien sûr donné lieu à des interrogations de notre part, et les réponses données ont toujours fait référence à des arguments rationnels : " le compagnonnage contribue à améliorer les capacités et les compétences de l'équipe. C'est de l'apprentissage en " peer to peer », puisque le coach et/ou l'évaluateur (la même personne) a eu le même vécu que la personne évaluée. La connaissance est transmise par des personnes qui avaient les mêmes missions il y a quelques années " (un associé). Un consultant junior est censé faire preuve de suffisamment d'autonomie pour construire puis proposer son travail avant qu'on ne le conseille. En l'occurrence, ce sont des associés désignés qui s'en chargent. "Pas de solution (personnelle), pas de conseil (personnalisé) » (un associé). Pour cette raison, les associés prennent toujours part aux missions, des premiers contacts commerciaux jusqu'à la post-évaluation.

Le compagnonnage n'est cependant pas qu'un mot pour traduire le processus d'accompagnement des nouveaux venus. II traduit aussi l'instauration d'un système traditionnel bien défini. 
L'absence d'écriture confrontée à la constance de la pratique constitue un premier élément cohérent avec le fonctionnement d'un système traditionnel. De surcroît, le formalisme, le recours aux références externes, et le processus d'accompagnement des nouveaux relèvent d'une référence permanente à une forme de tradition. A des scénarii d'action fondés sur l'agir rationnel en finalité, il faut opposer un vocabulaire et des mises en œuvre ancrés dans la rationalité en valeur et l'agir traditionnel (Weber, 1922).

Le tableau 2 dresse un parallèle entre les principales caractéristiques de l'induction pratiquée par le cabinet de conseil et celle mise en œuvre dans les organisations compagnonniques. A l'exception du potentiel de violence et du caractère secret qui marquent ces dernières, le rapprochement permet de représenter plus précisément l'ancrage du cabinet de conseil étudié dans les agir traditionnels et en valeur dont le compagnonnage constitue un archétype. L'inventaire des caractéristiques est proposé en première colonne. La seconde livre leur justification rationnelle (ostensive et légitimatrice). La troisième établit les similitudes avec le système compagnonnique. A la lecture de ce tableau, il apparaît clairement que l'usage du terme de " compagnonnage » dans le cabinet ne relève pas seulement d'une coquetterie discursive.

Processus d'induction (caractéristiques)

Interprétations archétypales

L'élitisme assumé (recrutement)

Trouver les meilleurs potentiels

Trouver les meilleures « pierres brutes »

La motivation est un critère pré-assuré

Le processus de recrutement est si lourd que seules des personnes motivées s'y engagent.

Certitude d'être suffisamment attractif et exigeant pour ne recevoir que des candidats motivés.

Les capacités plutôt que les connaissances techniques.

La technique s'apprend plus aisément sur le terrain.

Un candidat est une « pierre brute » qui doit révéler ses capacités à être taillée à la perfection plutôt qu'être grossièrement sculptée.

Les consultants et les associés participent au processus.

Accroître l'implication du personnel et promouvoir l'esprit d'équipe sont les objectifs poursuivis.

L'accueil des candidats et leur initiation requièrent l'approbation de la communauté dans son ensemble. La cooptation est le seul moyen de créer une communauté autour de valeurs partagées, et cela a toujours été le cas.

L'accompagnement et l'évaluation sont réalisés par des pairs. 
Efficience des processus de feedback et d'évaluation.

La communauté survit tant que chaque individu suit la même voie que ses prédécesseurs. Les maîtres montrent le voie de "l'ars perfecta ". Ils transmettent des valeurs, des rites, et le savoir faire.

Les consultants juniors doivent savoir proposer une solution avant d'être conseillés.

Promouvoir l'autonomie.

Les apprentis doivent « faire leur route » avant d'être guidés par le maître.

La règle du "Up or out"

Conserver les résultats au meilleur niveau possible.

Les apprentis deviennent compagnons (grade intermédiaire) une fois qu'ils ont prouvé leur capacité. Ceux qui n'y parviennent pas se voient offrir une seconde chance, ou sont exclus.

Une expérience variée dans un court délai.

Assurer la polyvalence et l'adaptabilité des consultants.

Le système du Tour de France favorise la rencontre de différents maîtres et le travail dans des contextes différents. II a valeur initiatique.

Un formalisme sans écrit.

Taille de l'entreprise et hiérarchie plate.

Tradition orale et rituelle.

La référence à d'autres cabinets.

Faire de même que « ce qui marche ailleurs ».

Les nouvelles entités sont fondées par d'anciens maîtres qui répliquent les règles existantes.

Rationalité en finalité

Rationalité en valeur - Agir traditionnel

Articulation moyens-fins

Archétype compagnonnique

Tableau 2 - L'induction dans le cabinet de conseil: ses caractéristiques, leur justification, et leur compréhension

Nombreux sont les principes du compagnonnage issus de considérations empiriques. Toutefois, ils sont aujourd'hui érigés en pures traditions.

\subsection{Au-delà du cas : propositions sur les dynamiques d'institutionnalisation d'une pratique}


La présente étude de cas laisse apparaître qu'une pratique peut acquérir le statut d'institution de manière autonome. En d'autres termes, elle s'intègre, par sa mise en œuvre, aux finalités d'ordre supérieur qui constituent ce que l'on peut appeler une institution (Pesqueux, 2007). La méthodologie de cette recherche - fondée sur l'analyse de l'interaction entre les aspects ostensifs, performatifs et les artefacts constitutifs de la pratique - permet de relever un ensemble de pistes et de questionnements de recherche sur les dynamiques d'institutionnalisation.

La présente étude confirme d'abord le caractère déterminant de la reproduction, qui relève de deux mécanismes distincts et complémentaires. II s'agit avant tout du mimétisme direct, c'est-à-dire la réitération de ce qui a été observé ou vécu. En second lieu, le discours traduit de manière précise la mise en œuvre, et revêt à son tour un caractère normatif pour les implémentations futures. D'aucuns diraient que, dans ce contexte, la pratique se mue en routine. Dans le cas du cabinet de conseil, l'induction n'est jamais réalisée comme une simple habitude. Elle relève plus précisément du geste rituel, ancré dans des convictions et une forme de réflexion. Les aspects ostensifs de la pratique sont là pour en témoigner. Ils se renouvellent en permanence par de nouveaux exemples et contrexemples alors que la référence (l'archétype visé) demeure la même.

La conjonction de l'autorité et de l'ancienneté contribue aussi à l'institutionnalisation, en cela qu'elle constitue une source de légitimité supplémentaire pour « tout ce qui s'est fait jusqu'à présent ». Elle recourt bien sûr au modèle cumulatif de l'expérience : plus on a de vécu, plus on est apte à diriger une mission, une démarche commerciale, le cabinet. Mais elle traduit aussi l'idée que l'ancienneté est la garantie d'une "répétition suffisante » des pratiques ritualisées : plus on a eu l'expérience de la même pratique (plus on l'a répétée), plus on est capable d'en assurer la reproduction (moins on est capable de la remettre en cause).

La dernière réflexion suscitée par cette recherche repose sur l'association de rationalités et d'agir contradictoires. Les scénarii d'action adoptés par le cabinet reposent sans exception sur la rationalité en finalité. Les actes accomplis de manière rituelle se réfèrent à l'agir traditionnel. Cette opposition est contenue dans la pratique d'induction étudiée, elle en est même constitutive. II serait facile d'interpréter cela sous l'angle d'une « hypocrisie », c'est-à-dire sous celui d'un discours rationnalisant conçu pour couvrir des actes d'une tout autre nature. Le procès en non-sincérité adressé aux aspects ostensifs relève d'une simplification qui ignore l'attachement (de longue date ${ }^{30}$ ) des membres de l'organisation à la rationalité téléologique. Ce hiatus est au contraire une clé pour comprendre le processus d'institutionnalisation d'une pratique dans une entreprise qui se conçoit comme un « temple » de la rationalité. Les éléments ostensifs légitiment une façon d'agir qui sans eux ne serait pas acceptable pour les membres de l'organisation. L'un des faits les plus marquants à cet égard est l'absence (dans les aspects performatifs) 
d'une rationalité adaptative qui dépasse le cadre de remises en cause minimes du mode fonctionnement établi. Aucune « réforme » de la pratique en question n'a jamais été envisagée. L'explication rationnelle produite pour ce phénomène est des plus simples : " on ne change pas ce qui marche ». La rationalité, probablement érigée en valeur (ie une fin en soi), fonde la tradition dans l'organisation. De la polémique assumée naît une forme d'harmonie qui n'appartient qu'à cette organisation, ce qui suggère que la raison contradictoire est en fin de compte plus structurante que l'aveuglement rationnel.

Cette étude met donc à jour trois dynamiques complémentaires qui conduisent à l'institutionnalisation de la pratique observée. La figure 3 en rappelle la nature, et les interactions observées : si une institution repose sur la répétition, elle relève aussi de processus de légitimation, assurés non seulement par la conjonction de l'ancienneté et de l'autorité, mais aussi (et plus fondamentalement) par l'opposition permanente entre la rationalité des scénarii d'action et le caractère traditionnel des façons d'agir.

Une coïncidence :

Une opposition :

autorité et ancienneté

rationalité téléologique et tradition

Un mécanisme :

la reproduction

Mimétisme simple Aller/retour discours- mise en œuvre

\section{Institutionnalisation}

Figure 4 : Trois composantes fondamentales des dynamiques d'institutionnalisation

\section{Conclusion}

Les sciences humaines ne sont pas capables d'élaborer une intelligibilité des phénomènes selon le modèle des sciences physiques (Liu, 1997).

L'intelligibilité pour le chercheur en sciences physiques repose sur la mise en évidence de causalités, alors que la complexité des phénomènes humains ne permet pas de les identifier clairement. De fait, le caractère diffus des causes possibles, les cas de récursivité, et la singularité du fait social (comme phénomène scientifique) excluent le développement d'une intelligibilité en sciences humaines comparable à celle recherchée en sciences physiques. Pour cette raison, le chercheur en sciences humaines confronte sa 
représentation (sa compréhension) du phénomène étudié à celle des autres, et c'est du débat scientifique que peut éventuellement émerger une représentation commune (si tant est que ce soit possible).

L'appréhension d'un phénomène dans sa complexité nécessite une étude approfondie de ce dernier. Dans ce contexte, la méthodologie d'approche des situations d'entreprise par l'étude de cas se justifie pleinement. Pour ce qui concerne cette recherche, ce sont bien des éléments relativement inattendus (au sens de Yin, 1994) qui ont émergé de la recherche. A l'attente d'un positionnement « intégralement » rationnel, nous nous sommes vu opposer la force d'un agir traditionnel et ancré dans des systèmes de valeur. De l'étude des facteurs de changement sur une pratique spécifique, nous avons dû appréhender des dynamiques conduisant à l'immuabilité d'un système. Nous aurions pu rencontrer des routines inertes, menées par la force de l'habitude et de la tradition, ou bien une pratique en constante évolution. Au lieu de cela, nous avons observé des interactions constantes et une forme globale immobile.

Cette étude apporte un éclairage particulier non pas sur le changement, mais sur la mutation d'une pratique en institution (Jardat et Méric, 2007). Si nous n'avons pas assisté directement à cette mutation, nous avons pu examiner comment les trois volets de la pratique (ostensifs, performatifs et artefacts) interagissent pour assurer cette institutionnalisation. II ressort de ce travail que le mimétisme et le rôle de l'ancienneté dans la formation de l'autorité constituent deux dynamiques fondamentales dans ce processus. Toutefois, dans un univers gestionnaire comme celui étudié, l'institutionnalisation n'apparaît possible que si l'organisation porte, dans son discours et ses actes deux modèles a priori contradictoires. L'archétype traditionnel alimente le phénomène de reproduction, et l'archétype de la rationalité téléologique constitue la seule source possible de légitimité dans l'action. Sans l'un des pendants de cette polémique, la situation deviendrait insupportable pour la plupart des acteurs - absence de sens dans un cas, carence en repères stables dans l'autre. Des études analogues dans d'autres organisations devraient permettre d'enrichir ou nuancer ces résultats, si du moins l'on s'intéresse à la pratique d'induction. Cette dernière présente en effet l'intérêt de constituer un processus d'institutionnalisation majeur, en même temps qu'elle peut devenir à son tour une partie intégrante de l'institution.

\section{Bibliographie}

Bourdieu P. (1977) Outline of a Theory of Practice, Cambridge University Press, New York.

Bourdieu P. (1990) The Logic of Practice, Stanford University Press, Stanford. Castoriadis C. (1998) The Imaginary Institution of Society, trans. K. Blamey, the MIT Press, Boston. Cyert R.M., March J.G. (1963) A Behavioral Theory of the Firm, Prentice Hall, Englewood Cliffs.

Eisenhardt, K., 1989, "Building Theories from Case Study Research", Academy of 
Management Review, vol.14 n4, pp.532-550. Feldman M.S. (2000) "Organizational Routines as a source of continuous change", Organization Science, vol. 11, pp. 661629.

Feldman M.S., Pentland B.T. (2003) "Reconceptualizing organizational routines as a source of flexibility and change", Administrative Science Quarterly, N.48, pp. 94-118. Feldman M.S. (2003) "A performative perspective on stability and change in organizational routines", Industrial and Corporate Change, vol. 12 , n ${ }^{\circ}$, pp.727-752. Fiol M. (1991) La Convergence des buts dans l'entreprise, thèse de Doctorat d'Etat, Université Paris IX Dauphine.

Giddens A. (1984) The Constitution of Society, University of California Press, Berkeley. Glaser B.G., Strauss A.L. (1967), The Discovery of Grounded Theory: Strategies for Qualitative Research, Aldine de Gruyter, New York. Jardat R., Méric, J. (2007), "Induction as an institutionalized and institutionalizing practice - retail banking and consultancy in France", AIM GNOSIS International Conference, London, 5-7 September

Latour B. (1987) Science in Action. How to Follow Scientists and Engineers through Society, Harvard University Press, Cambridge. Levitt B. March J.G. (1988) 'Organizational learning', Annual Review of Sociology, vol. 14, pp.314-340. Liu, M. (1997) Fondements et pratiques de la recherche action, L'Harmattan, Paris. March J.G., Simon H.A. (1958) Organizations, Wiley, New York. Miner A. (1990) "Structural evolution through idiosyncratic jobs: the potential for unplanned learning", Organization Science, vol. 1, pp.195-210.

Nonaka I., Takeuchi H. (1995) The Knowledge Creating Company. How the Japanese Companies Create the Dynamics, Oxford University Press, Oxford. Pentland B.T., Feldman M.S. (2005) "Organizational routines as a unit of analysis", Industrial and Corporate Change, Vol. 14, N.5, pp. 793-815.

Pesqueux Y. (2007) Gouvernance et privatisation, PUF, Paris.

Strauss A.L., Corbin J. (1990), Basics of Qualitative Research: Grounded Theory Procedures and Technics, Sage, Newbury Park. Weber M. (1922) Wirtschaft und Gesellschaft, Grundriß des Verstehender Soziologie, Mohr Siebeck, Erfurt.

Wenger E., Snyder W. (2000) "Communities of practice: the organizational frontier“, Harvard Business Review, January-February, pp. 139-145 Yin R.K. (1994) Case Study Research : Design and Methods, $2{ }^{\text {nd }}$ Edition, Sage, California. Zollo M., Winter S.G. (2002) "Deliberate learning and the evolution of dynamic capabilities", Organization Science, vol. 13, n³, pp.339-351 